\title{
Higher Starting Dose of Ciclosporin Optimized Therapeutic Levels in Patients Receiving Phenytoin for Busulfan-induced Seizure Prophylaxis
}

\author{
Ying Shi $\mathrm{Ho}^{1}$, Mui Fong Chong', Vin $\mathrm{Cci} \mathrm{Ng}{ }^{1}$, Aloysius $\mathrm{Ho}^{2}$, Hong Yen Ng${ }^{1}$ \\ 'Department of Pharmacy, Singapore General Hospital, Singapore, ${ }^{2}$ Department of Hematology, Singapore General \\ Hospital, Singapore
}

\begin{abstract}
Background: Despite understanding the drug-drug interaction between phenytoin and ciclosporin (CsA), there is no recommended CSA dosing in patients receiving phenytoin as seizure prophylaxis in busulfan-based conditioning regimens. This drug-drug interaction has resulted in patients with sub-therapeutic levels at day 0 (D0) of allogeneic hematopoietic stem cell transplantation (alloHSCT) and at risk for acute graft-versus-host disease (aGVHD).

Objective/Methods: A single-center historical-control study was conducted at Singapore General Hospital between March 2010 and July 2019 to evaluate a new dosing strategy. Patients with phenytoin received a higher starting dose of intravenous CsA ( $4 \mathrm{mg} / \mathrm{kg} /$ dose twice daily instead of $3 \mathrm{mg} / \mathrm{kg} /$ dose twice daily). The primary endpoint of this study was to determine the proportion of patients with therapeutic CsA levels at D0. Secondary endpoints included median CsA level on D-1 and D0, time to the therapeutic target, incidence and severity of aGVHD, and safety profile.

Results: A total of 91 patients were included in this study. Patients with therapeutic CsA at D0 was higher $(66.7 \%)$ in the study arm than in the control arm $(24.7 \%)(p=0.006)$. The median CsA concentration at D0 in the study arm was $284 \mathrm{ng} / \mathrm{mL}$ (range, 144-441 ng/mL) as compared to the control arm, $255 \mathrm{ng} / \mathrm{mL}$ (range, 104580). There was no difference in the time to therapeutic range and the cumulative incidence of aGVHD. There were no significant differences in the safety outcomes.

Conclusion: The new strategy with higher dosing based on the actual body weight should be adopted as it resulted in a higher proportion of patients with therapeutic CsA at D0, without an increase in CsA-related adverse events.
\end{abstract}

Key words drug interactions, ciclosporin, phenytoin, busulfan, graft-versus-host-disease

Submitted December 20, 2020; Accepted March 31, 2021; Published online August 25, 2021; Issued online August 25, 2021

Correspondence: Ying Shi Ho, Department of Pharmacy Singapore General Hospital, 1 Hospital Drive Singapore 169608, Block 7 Level 2 Hematology Pharmacy, Singapore, E-mail: ho.ying.shi@sgh.com.sg

\section{Introduction}

Acute graft-versus-host disease (aGVHD) is one of the most common complications after allogeneic hematopoietic stem cell transplantation. It is known to cause significant morbidity and mortality in transplant recipients ${ }^{1}$. The development of aGVHD is primarily T-cell mediated, and it occurs when the immune cells from the donor graft recognize the tissues of the recipient as foreign, thereby triggering an immune response that causes injury to the host tissue and organs ${ }^{2}$. Despite current prophylactic strategies, patients remain at risk for developing aGVHD ${ }^{3}$. The prevalence of aGVHD ranges from approximately $45 \%$ in patients with matched related donor grafts to up to $80 \%$ in patients with one antigen mismatched unrelated donor graft ${ }^{4}$.

Sub-therapeutic levels of ciclosporin (CsA) at the initial phase of alloHSCT may increase the risk of aGVHD and early graft failure. It has been postulated that the activation and expansion of alloreactive $\mathrm{T}$ cells usually occur early after graft infusion ${ }^{5}$. Therefore, achieving therapeutic levels by the time of stem cell infusion, denoted as Day 0 (D0), would be potentially protective against aGVHD. Previous studies have identi- 
fied that early therapeutic CsA levels are strongly correlated with the incidence and severity of aGVHD ${ }^{5-8}$.

Based on a retrospective study conducted by Ho et al. in Singapore General Hospital (SGH) between March 2010 and December 2015, only 25.4\% of patients who received phenytoin as seizure prophylaxis had therapeutic CsA at D0 of alloHSCT. These patients also required higher CsA doses and a longer time to achieve the therapeutic level. A new CsA dosing guide was derived from the results of this study'. In addition, total body weight (TBW) for CsA dosing was also implemented according to the recommendation from the Acute Leukemia Working Party of the European Society for Blood and Marrow Transplantation (EBMT) ${ }^{10}$.

This study aimed to evaluate the impact of revised CsA dosing on transplant outcomes. The major revisions made to CsA dosing included 1) a new starting dose regimen for CsA and 2) use of TBW instead of adjusted body weight (ABW) for obese patients. The primary endpoint of the study was to determine the proportion of patients with therapeutic CsA levels by D0 between the study and control arms. Secondary endpoints included median CsA levels at Day -1 (D-1) and on D0, time to the therapeutic target, incidence and severity of acute graft-versus-host disease (aGVHD), and safety profile associated with the new dosing strategy.

\section{Materials and Methods}

\section{Study design and inclusion/exclusion criteria}

This was a historical-control single-center study at a tertiary care general hospital, which included patients who underwent alloHSCT at the Singapore General Hospital (SGH). The study arm consisted of patients from August 2018 to April 2019, while the control arm included patients from March 2010 to December 2015. Only patients who received CsA as GVHD prophylaxis and had phenytoin as seizure prophylaxis in the busulfan-based conditioning regimen were included. Patients who had prior alloHSCT, received tacrolimus as initial GVHD prophylaxis, lost to follow-up, or had incomplete data entry were excluded.

\section{Data collection and management}

Patient, transplant, and GVHD variables were collected through inpatient and outpatient medical record reviews. The collected study data were managed using REDCap electronic data capture tools hosted at $\mathrm{SGH}^{11}$. This study was approved by the SingHealth Centralized Institutional Review Board (CIRB), conducted in accordance with the ethical principles of the Declaration of Helsinki, and consistent with the Singapore Good Clinical Practice guidelines. A waiver of informed consent was obtained from all patients.

\section{Treatment}

The transplant conditioning regimen was determined based on various factors, including age, comorbidities, disease indication for transplant, type of donor (sibling vs. unrelated), degree of matching, disease status, and stem cell source. Busulfan was administered around Day -7 of alloHSCT for 2-4 days, depending on the intensity of the conditioning regimen. Patients also received oral (PO) phenytoin $300 \mathrm{mg}$ starting on the day before the first busulfan dose and continued for 24 hours after the last busulfan dose. Blood concentrations of phenytoin were not measured at our institution. All patients received CsA (Sandimmune ${ }^{\circledR}$ ) as GVHD prophylaxis, starting on Day -3 of alloHSCT. In the study arm, patients with phenytoin as seizure prophylaxis received a higher starting dose of intravenous (IV) CsA 4 $\mathrm{mg} / \mathrm{kg} /$ dose twice daily compared to IV CsA $3 \mathrm{mg} / \mathrm{kg} /$ dose twice daily.

Recipients of myeloablative (MA) alloHSCT also received methotrexate (MTX) intravenous bolus $15 \mathrm{mg} / \mathrm{m}^{2}$ on Day +1 and $10 \mathrm{mg} / \mathrm{m}^{2}$ Day $+3,+6$, and +11 as part of GVHD prophylaxis, whereas recipients of reducedtoxicity conditioning (RTC) and non-myeloablative (NMA) alloHSCT received either mycophenolic acid (MPA; Myfortic) orally $720 \mathrm{mg}$ twice daily starting on day 0 or MTX intravenous bolus $5 \mathrm{mg} / \mathrm{m}^{2}$ on days +1 , +3 , and +6 . MTX doses were attenuated or omitted in patients with severe mucositis or renal and hepatic dysfunction. All patients with unrelated donors also received anti-thymocyte globulin (Thymoglobulin ${ }^{\circledR}$, rabbit-derived ATG) intravenously $0.5 \mathrm{mg} / \mathrm{kg}$ for 1 day followed by $2 \mathrm{mg} / \mathrm{kg}$ for 2 days, as GVHD prophylaxis. In the absence of GVHD symptoms, MPA and CsA were tapered per transplant protocol. Supportive care in terms of antibacterial, antiviral, and antifungal prophylaxis, blood products, and nutritional care were provided as per institutional standard procedures. As part of our transplant antifungal prophylaxis, patients were initiated on oral posaconazole (a strong CYP3A4 inhibitor) after D0 of alloHSCT.

\section{Definitions}

Early therapeutic CsA was defined as CsA level of 250-400 ng/mL for the first four weeks of alloHSCT, starting from the day of stem cell infusion (D0). Thereafter, the therapeutic target for CsA was individualized and subjected to donor source or human leukocyte antigen (HLA) disparity and physicians' discretion. The clinical diagnosis of aGVHD was defined according to the National Institutes of Health (NIH) consensus criteria in this study (Table 1) ${ }^{12,13}$. Obesity was defined as total body weight (TBW) more than $130 \%$ of ideal body weight (IBW) $)^{9}$. 
Table 1. Classification of GVHD as per NIH consensus criteria

\begin{tabular}{lccc}
\hline Category of aGVHD & $\begin{array}{c}\text { Time of symp- } \\
\text { toms after HSCT }\end{array}$ & $\begin{array}{c}\text { Presence of } \\
\text { aGVHD features }\end{array}$ & $\begin{array}{c}\text { Presence of } \\
\text { cGVHD features }\end{array}$ \\
\hline Hyperacute, early onset acute & $\leq 14$ days & Yes & No \\
$\begin{array}{l}\text { Classic acute } \\
\begin{array}{l}\text { Persistent acute, recurrent acute, } \\
\text { late-onset acute }\end{array}\end{array}$ & $\leq 100$ days & Yes & No \\
\hline $\begin{array}{l}\text { GVHD, graft versus host disease; } \mathrm{NIH} \text {, national institutes of health; aGVHD, acute graft versus host } \\
\text { disease; HSCT, hematopoietic stem cell transplantation; cGVHD, chronic graft versus host disease }\end{array}$
\end{tabular}

\section{Assessment of CsA levels}

Ciclosporin trough levels were monitored at least twice weekly during the first four weeks of alloHSCT, starting three days after the initiation of CsA. Patients with sub-therapeutic CsA levels were monitored more frequently, as indicated clinically. CsA doses were then titrated to reach the target concentrations. Whole blood samples were collected and measured using chemiluminescent microparticle (CMIA) immunoassay (Abbott Laboratories: ARCHITECT Cyclosporine assay i1000 ARCHITECT platform).

\section{Study endpoints}

The primary endpoint of the study was to determine the proportion of patients with therapeutic CsA levels at D0 between the study and control arms.

The secondary endpoints were to evaluate 1) median CsA level on D-1 and D0, 2) time to the therapeutic target, 3) incidence and severity of aGVHD, and 4) safety profile associated with the new dosing strategy.

\section{Statistical analysis}

All data collected were analyzed using the Statistical Package for the Social Sciences (SPSS version 25.0). Categorical variables were computed using Chi-square, Fisher's exact test, or Kruskal-Wallis test, and continuous variables using the Mann-Whitney U test. Statistical significance was set at $P<0.05$.

\section{Results}

\section{Patient, graft and transplant characteristics}

In this analysis, 12 patients were included in the study arm and 79 in the control arm. The characteristics of the patients are summarized in Table 2 . The baseline patient, graft, and transplant characteristics were largely comparable in both arms. The median age was 42.5 years (range, 18-58) and 46 years (range, 12-68) in the study and control arms, respectively. The majority of patients were diagnosed with acute leukemia. The most common stem cell source utilized in our transplant center was peripheral blood. Notably, the study arm had a relatively shorter median follow-up at 4.8 months (range, 0-11.5), while the control arm had a median follow-up of 49 months (range, 10-91 months).

\section{Achievement of therapeutic CsA levels}

The proportion of patients with therapeutic CsA on D-1 were $25 \%$ (3 patients) and $7.8 \%$ (6 patients) in the study and control arm respectively (Table 3). A higher proportion of patients achieved therapeutic CsA on D0 in the study arm at $66.7 \%$ (eight patients) than $24.7 \%$ (19 patients) in the control arm. The median duration to achieve therapeutic CsA was 4 days (range, 3-7 days) and 5 days (range, 2-15 days) in the study and control arms, respectively. In the study and control arms, the median CsA concentrations on D-1 were subtherapeutic at $225.5 \mathrm{ng} / \mathrm{mL}$ (range, $78-389 \mathrm{ng} / \mathrm{mL}$ ) and $196 \mathrm{ng} / \mathrm{mL}$ (range, 46-701 ng/mL), respectively. However, it was noted that in both arms, the median CsA concentrations improved to therapeutic levels on D0 at $284 \mathrm{ng} / \mathrm{mL}$ (range, 144-441) and $255 \mathrm{ng} / \mathrm{mL}$ (range, 104-580), respectively. In addition, it was observed that the range of CsA concentration in the control arm was much wider across all dosing groups than in the study arm.

\section{Incidence and severity of aGVHD}

The cumulative incidence of all grades of aGVHD was $8.3 \%$ and $53.2 \%$ in the study and control arms, respectively (Table 4). The majority of the patients in the control group had classic aGVHD (78.6\%). Nine patients $(21.4 \%)$ in the control arm had late-onset aGVHD, which developed beyond the traditional 100 days cut-off after HSCT. Given the short follow-up period of 4.8 months in the study arm, information on late-onset aGVHD was not available at the time of data analysis.

\section{Safety of CsA}

Two $(16.7 \%)$ patients in the study arm and 25 patients (31.6\%) in the control arm experienced adverse events with CsA. The types of adverse events are summarized in Table 5. All patients required cessation of CsA therapy and were substituted with tacrolimus. No fatalities resulted from CsA toxicity in the study and control arms. 
Table 2. Baseline Patient, Graft and Transplant Characteristics

\begin{tabular}{|c|c|c|}
\hline Characteristics & Study Arm $(n=12)$ & Control Arm $(n=79)$ \\
\hline Median age, years (range) & $42.5(18-58)$ & $46(12-68)$ \\
\hline Male gender, n (\%) & $6(50)$ & $37(46.8)$ \\
\hline $\begin{array}{l}\text { Race, } \mathrm{n}(\%) \\
\text { Chinese } \\
\text { Malay } \\
\text { Indian } \\
\text { Others }\end{array}$ & $\begin{array}{l}9(75) \\
1(7.7) \\
2(16.7) \\
0(0)\end{array}$ & $\begin{aligned} 59 & (74.7) \\
6 & (7.6) \\
3 & (3.8) \\
11 & (13.9)\end{aligned}$ \\
\hline $\begin{array}{l}\text { Disease, } \mathrm{n}(\%) \\
\text { ALL } \\
\text { AML } \\
\text { MDS/ MF/ CML } \\
\text { Lymphoma }\end{array}$ & $\begin{array}{l}1(8.3) \\
5(41.7) \\
6(50) \\
0\end{array}$ & $\begin{aligned} 9 & (11.4) \\
47 & (59.5) \\
20 & (25.3) \\
3 & (3.8)\end{aligned}$ \\
\hline $\begin{array}{l}\text { CMV serostatus, n (\%) } \\
\text { Seropositive patients }\end{array}$ & $8(66.7)$ & $55(69.6)$ \\
\hline $\begin{array}{l}\text { Donor type, } \mathrm{n}(\%) \\
\text { Matched related donor (MRD) } \\
\text { Matched unrelated donor (MUD) } \\
\text { Haploidentical/ Cord/ Others }\end{array}$ & $\begin{array}{l}7(58.3) \\
5(41.7) \\
0(0)\end{array}$ & $\begin{array}{r}50(63.3) \\
20(25.3) \\
9(11.4)\end{array}$ \\
\hline $\begin{array}{l}\text { Stem cell source, } \mathrm{n}(\%) \\
\text { Peripheral blood } \\
\text { Bone marrow } \\
\text { Cord blood }\end{array}$ & $\begin{array}{c}12(100) \\
0(0) \\
0(0)\end{array}$ & $\begin{array}{c}76(96.2) \\
3(3.8) \\
0(0)\end{array}$ \\
\hline $\begin{array}{l}\text { Conditioning, n (\%) } \\
\text { Myeloablative (MA) } \\
\text { Non-myeloablative (NMA) } \\
\text { Reduced-toxicity (RTC) }\end{array}$ & $\begin{array}{l}6(50) \\
0(0) \\
6(50)\end{array}$ & $\begin{array}{l}36(45.6) \\
32(40) \\
11(13.9)\end{array}$ \\
\hline $\begin{array}{l}\text { GVHD prophylaxis, } n \text { (\%) } \\
\text { CsA/MTX } \\
\text { CSA/MPA } \\
\text { CsA/MTX/MPA } \\
\text { ATG-containing (rabbit-derived) }\end{array}$ & $\begin{array}{l}7(58.3) \\
5(41.7) \\
0(0) \\
6(50)\end{array}$ & $\begin{aligned} 65 & (82.3) \\
19 & (24.1) \\
5 & (6.3) \\
35 & (44.3)\end{aligned}$ \\
\hline Obese patients, $\mathrm{n}(\%)$ & 7 (58.3) & $12(15.2)$ \\
\hline Median follow up of survivors, months (range) & $4.8(0-11.5)$ & $49(10-91)$ \\
\hline
\end{tabular}

Table 3. Characterization of CsA Levels

\begin{tabular}{|c|c|c|c|c|}
\hline Characteristics & & Study Arm $(n=12)$ & Control Arm ( $n=79)$ & P-value \\
\hline \multirow{4}{*}{ On Day -1 } & Sub-therapeutic CsA concentration, n (\%) & $8(66.7)$ & $59(76.6)$ & 0.481 \\
\hline & Therapeutic CsA concentration, $\mathrm{n}(\%)$ & $3(25)$ & $6(7.8)$ & 0.09 \\
\hline & Supra-therapeutic CsA concentration, $\mathrm{n}(\%)$ & $1(8.3)$ & $12(15.6)$ & \\
\hline & Median CsA concentration, $\mathrm{ng} / \mathrm{mL}$ (range) & $225.5(78-389)$ & $196(46-701)$ & 0.532 \\
\hline \multirow{4}{*}{ On Day 0} & Sub-therapeutic CsA concentration, $\mathrm{n}(\%)$ & $2(16.7)$ & $37(48.1)$ & 0.724 \\
\hline & Therapeutic CsA concentration, $\mathrm{n}(\%)$ & $8(66.7)$ & $19(24.7)$ & 0.006 \\
\hline & Supra-therapeutic CsA concentration, n (\%) & $2(16.7)$ & 21 (27.3) & 0.06 \\
\hline & Median CsA concentration, $\mathrm{ng} / \mathrm{mL}$ (range) & $284(144-441)$ & $255(104-580)$ & 0.343 \\
\hline \multicolumn{2}{|c|}{ Median days to achieve therapeutic CsA, days (range) } & $4(3-7)$ & $5(2-15)$ & 0.031 \\
\hline
\end{tabular}

\section{Discussion}

This study evaluated the impact of revised CsA dosing on CsA levels, aGVHD rates, and safety. The impact of TBW dosing has not been studied. A higher proportion of patients dosed based on the revised CsA dosing achieved therapeutic levels by D0 at $66.7 \%$ compared to $24.7 \%$ in the control arm. This difference reached statistical significance $(P=0.006)$, and an encouraging trend where the percentage of therapeutic levels at this important time-point was doubled. In addition, a lower proportion of patients had sub-therapeutic 
Table 4. Incidence and Severity of aGVHD

\begin{tabular}{|c|c|c|c|}
\hline & $\begin{array}{l}\text { Study Arm } \\
(\mathrm{n}=12)\end{array}$ & $\begin{array}{c}\text { Control Arm } \\
(n=79)\end{array}$ & P-value \\
\hline Diagnosis of aGVHD, n (\%) & $1(8.3)$ & $42(53.2)$ & 0.004 \\
\hline Classic aGVHD, n (\%) & $1(100)$ & $33(78.6)$ & - \\
\hline Late-onset aGVHD, n (\%) & $0(0)$ & $9(21.4)$ & - \\
\hline Day of onset of aGVHD, median (range) & $24\left(N^{\star}\right)$ & $42(19-246)$ & 0.233 \\
\hline Gastrointestinal GVHD, n (\%) & $1(100)$ & $9(21.4)$ & - \\
\hline Liver GVHD, n (\%) & $0(0)$ & $12(28.6)$ & - \\
\hline Skin GVHD, n (\%) & $0(0)$ & $16(38.1)$ & - \\
\hline Grade I, n (\%) & $1(100)$ & 5 (11.9) & - \\
\hline Grade II, n (\%) & $0(0)$ & $25(59.5)$ & - \\
\hline Grade III, n (\%) & $0(0)$ & $10(12.7)$ & - \\
\hline Grade IV, n (\%) & $0(0)$ & $2(2.5)$ & - \\
\hline
\end{tabular}

Table 5. Adverse Events Associated with CsA

\begin{tabular}{|c|c|c|c|}
\hline Characteristics & Study Arm ( $n=12)$ & Control Arm $(n=79)$ & P-value \\
\hline Any toxicity, n (\%) & $2(16.7)$ & $25(31.6)$ & 0.49 \\
\hline Gastrointestinal, n (\%) & $0(0)$ & $6(7.6)$ & - \\
\hline Nephrotoxicity, n (\%) & $0(0)$ & $9(11.4)$ & - \\
\hline Hypertension, n (\%) & $1(0.1)$ & $6(7.6)$ & - \\
\hline Thrombotic microangiopathy, $\mathrm{n}(\%)$ & $0(0)$ & $5(6.3)$ & - \\
\hline Others, n (\%) & Gum hyperplasia - $1(0.1)$ & $\begin{array}{c}\text { Headache }-2(2.5) \\
\text { Hyperbilirubinemia - } 3(3.8) \\
\text { Hypertriglyceridemia - } 1(0.1)\end{array}$ & - \\
\hline
\end{tabular}

CsA, ciclosporin

levels on D0 in the study arm than in the controls (16.7\% vs. $48.1 \%$ ). Low CsA levels on the day of stem cell infusion have implications for potential graft rejection. Compared to the controls, a significantly higher proportion of supra-therapeutic levels on D0 in the study arm was also observed (16.7\% vs. $27.3 \%)$, but this did not translate to increased adverse effects. The incidence and severity of aGVHD and toxicity associated with revised dosing of CsA were lower than those of the controls. Another promising trend observed was the narrowing of the median CsA levels. Although the median CsA levels on both D-1 and D0 were comparable between the study and control groups, the range of levels seemed narrower. The upper end of the range at D0 was $580 \mathrm{ng} / \mathrm{mL}$ in the control arm, which exceeded the toxic limit (approximately $500 \mathrm{ng} / \mathrm{mL}$ ) compared to $441 \mathrm{ng} / \mathrm{mL}$ in the study arm. This implied that besides optimizing weight-based dosing of CsA, therapeutic drug monitoring of CsA was also subjected to interpatient variability, and possibly an element of genetic polymorphism $^{14}$.

Overall, the results of this study were encouraging and substantiated a desired outcome, as previous studies have shown that early therapeutic CsA levels correlate with the development of $\mathrm{aGVHD}^{5,6,9}$. Ho et al. found that week 1 median CsA level <220 ng/mL (OR, 4.74; 95\% CI, 1.37-16.6; $P=0.015$ ) was a key risk factor for grade III-IV aGVHD ${ }^{9}$. Malard et al. reported that early and high therapeutic CsA levels were strongly correlated with the incidence and severity of aGVHD ${ }^{6}$. They found that lower CsA levels in the first two weeks post alloHSCT had a significantly higher risk of grade III-IV aGVHD. They identified CsA concentration <348 ng/ $\mathrm{mL}$ in the first week following graft infusion as the strongest parameter to increase the risk of grade III-IV aGVHD (OR: 4.16, 95\% CI: 0.08-0.73, $P=0.012$ ). Similarly, Rogosheske et al. demonstrated that CsA levels $<200 \mathrm{ng} / \mathrm{mL}$ were associated with a higher risk of $\mathrm{aGVHD}^{5}$. While therapeutic CsA levels at Day 0 may not translate into therapeutic levels in the first few weeks of alloHSCT, it provided a good starting point to achieve and maintain therapeutic levels at the initial phase of alloHSCT. This was supported by the shorter time to therapeutic range in the study arm $(P=0.07)$.

Maintaining immunosuppressive drugs, such as CsA, within desired levels is not an easy task given its nar- 
row therapeutic window ${ }^{15}$. As a CYP3A4 substrate, drug-drug interactions with CYA3A4 inducers and inhibitors may significantly affect CsA levels, requiring pre-emptive management and close monitoring. Phenytoin, a strong CYP3AS4 inducer, forms the backbone of busulfan-induced seizure prophylaxis in alloHSCT recipients. Despite understanding the drug-drug interaction between phenytoin and CsA, there is no recommended CsA dosing in patients receiving phenytoin as seizure prophylaxis in busulfan-based conditioning regimens. To our knowledge, this is the first study to propose a higher IV CsA dose when used in combination with phenytoin as seizure prophylaxis. Our data also showed that the new dosing strategy adopted for CsA is safe and does not increase the toxicity associated with CsA.

Other strategies to avoid this significant drug-drug interaction have been adopted by other transplant centers, including the use of non-interacting anti-epileptic medicines (AEMs) such as levetiracetam ${ }^{16-19}$. AEMs are known to be effective in preventing busulfan-induced seizures; however, it remains unclear if this strategy affects outcomes ${ }^{20}$. Previous reports were not sufficient to evaluate whether relapse or non-relapse mortality (NRM) and overall survival (OS) might be affected by alternative AEMs compared to phenytoin in patients treated with busulfan-based conditioning regimens $^{18,19,21,22}$, In a study by Mccune et al., there was no difference in the outcomes for NRM and $\mathrm{OS}^{23}$. Hence, clinicians should consider alternative AEMs, such as levetiracetam, as the drug of choice for seizure prophylaxis, given its favorable drug interaction profile. However, the acceptance of alternative AEMs to prevent busulfan-induced seizures in alloHSCT recipients has been slow ${ }^{23}$. This strategy of adopting alternative AEMs is likely more feasible in institutions that have the facility to support busulfan therapeutic drug monitoring (TDM) to ensure that patients receive the intended busulfan plasma area under the curve $\mathrm{e}^{24}$. As our institution currently does not support busulfan TDM, phenytoin will continue to be the drug of choice for busulfaninduced seizure prophylaxis, while alternative AEMs may be used with caution if the use of phenytoin is contraindicated.

This study provides valuable information on CsA dosing regimens in Asian patients receiving alloHSCT and phenytoin as seizure prophylaxis. However, this is not without limitations. First, the small sample size is a major drawback, yet this is expected given the nature of the study involving patients receiving alloHSCT. Second, it is prudent to have a longer follow-up period to truly ascertain the incidence and severity of aGVHD, which may occur beyond the typical 100-day cut-off. Lastly, the retrospective nature of the study is subject to biases and depends very heavily on the accurate charting of patients' symptoms in the clinical notes for the appropriate classification of aGVHD. We minimized subjectivity in the diagnosis of aGVHD by confirming the clinical symptoms with biopsy results, although it is not necessary for the diagnosis of aGVHD.

\section{Conclusion}

The revised dosing strategy of CsA is currently adopted in our clinical practice as a standard of care at $\mathrm{SGH}$. The dosing revision included a higher starting dose regimen for $\mathrm{CsA}$ and the use of total body weight instead of adjusted body weight. The new dosing strategy resulted in a higher proportion of patients with therapeutic CsA by day 0. There was no difference in the time to the therapeutic range and the cumulative incidence of aGVHD. Moreover, no meaningful differences in the safety outcomes were found.

\section{Author Contributions}

YSH and MFC performed the research, YSH, AH and HYN designed the research study, YSH and VCN analyzed the data and YSH wrote the paper.

\section{Conflicts of Interest}

The authors declare no conflict of interest. Disclosure forms provided by the authors are available on the website.

\section{References}

1. Appelbaum FR. The current status of hematopoietic cell transplantation. Annu Rev Med. 2003; 54: 491-512.

2. Hill GR, Ferrara JLM. The primacy of the gastrointestinal tract as a target organ of acute graft-versus-host disease: rationale for the use of cytokine shields in allogeneic bone marrow transplantation. Blood. 2000; 95: 2754-9.

3. Choi SW, Reddy P. Current and emerging strategies for the prevention of graft-versus-host disease. Nat Rev Clin Oncol. 2014; 11: 536-47.

4. Loiseau P, Busson M, Balere ML, Dormoy A, Bignon JD, Gagne K, et al. HLA Association with hematopoietic stem cell transplantation outcome: the number of mismatches at HLA-A, -B, -C, -DRB1, or -DQB1 is strongly associated with overall survival. Biol Blood Marrow Transplant. 2007; 13: $965-74$.

5. Rogosheske JR, Fargen AD, DeFor TE, Warlick E, Arora M, Blazar BR, et al. Higher therapeutic CsA levels earlypost transp lantation reduce risk of acute GVHD and improves survival. Bone Marrow Transplant. 2014; 49: 122-5.

6. Malard F, Szydlo RM, Brissot E, Chevallier P, Guillaume T, Delaunay $\mathrm{J}$, et al. Impact of cyclosporine-A concentration on the incidence of severe acute graft-versus-host disease after 
allogeneic stem cell transplantation. Biol Blood Marrow Transplant. 2010; 16: 28-34.

7. Punnett A, Sung L, Price V, Das P, Diezi M, Doyle J, et al. Achievement of target cyclosporine concentrations as a predictor of severe acute graft versus host disease in children undergoing hematopoietic stem cell transplantation and receiving cyclosporine and methotrexate prophylaxis. Ther Drug Monit. 2007; 29: 750-7.

8. Zeighami S, Hadjibabaie M, Ashouri A, Sarayani A, Khoee $\mathrm{SH}$, Mousavi S, et al. Assessment of cyclosporine serum concentrations on the incidence of acute graft versus host disease post hematopoietic stem cell transplantation. Iran J Pharm Res. 2014; 13: 305-12.

9. Ho YS, Ong PS, Chong MF, Ng VC, Ng HY. Early cyclosporine levels influence the incidence and severity of acute graft versus host disease in Asian patients receiving allogeneic haematopoietic stem cell transplant. [Abstract]. SGH 22 nd Annual Scientific Meeting, 2017. Abstract NC-P-AH-007.

10. Shem-Tov N, Labopin M, Moukhtari L, Ciceri F, Esteve J, Giebel S, et al. Chemotherapy dose adjustment for obese patients undergoing hematopoietic stem cell transplantation: a survey on behalf of the Acute Leukemia Working Party of the European Society for Blood and Marrow Transplantation. Oncologist. 2015; 20: 50-5.

11. Harris PA, Taylor R, Thielke R, Payne J, Gonzalez N, Conde JG. Research electronic data capture (REDCap)-- a metadatadriven methodology and workflow process for providing translational research informatics support. J Biomed Inform. 2009; 42: 377-81.

12. Vigorito AC, Campregher PV, Storer BE, Carpenter PA, Moravec CK, Kiem HP, et al. Evaluation of NIH consensus criteria for classification of late acute and chronic GVHD. Blood. 2009; 114: 702-8.

13. Saliba RM, de Lima M, Giralt S, Andersson B, Khouri IF, Hosing C, et al. Hyperacute GVHD: risk factors, outcomes and clinical implications. Blood. 2007; 109: 2751-8.

14. Hesselink DA, van Schaik RHN, van der Heiden IP, van der Werf M, Smak Gregoor PJH, Lindemans J, et al. Genetic polymorphisms of the CYP3A4, CYP3A5, and MDR-1 genes and pharmacokinetics of the calcineurin inhibitors cyclosporine and tacrolimus. Clin Pharmacol Ther. 2003; 74: 245-54.

15. Correa PM, Zuckermann J, Fischer GB, Castro MS. Immunosuppressive serum levels in allogeneic hematopoietic stem cell transplantation: pharmaceutical care contribution. Pharm
Pract (Granada). 2016; 14: 683.

16. Vassal G, Deroussent A, Hartmann O, Challine D, Benhamou E, Couanet DV, et al. Dose-dependent neurotoxicity of highdose busulfan in children: a clinical and pharmacological study. Cancer Res. 1990; 50: 6203-7.

17. Murphy CP, Harden EA, Thompson JM. Generalized seizures secondary to high-dose busulfan therapy. Ann Pharmacother. 1992; 26: 30-1.

18. Eberly AL, Anderson GD, Bubalo JS, McCune JS. Optimal prevention of seizures induced by high-dose busulfan. Pharmacotherapy. 2008; 28: 1502-10.

19. Mielcarek M, Furlong T, O'Donnell PV, Storer BE, McCune JS, Storb R, et al. Posttransplantation cyclophosphamide for prevention of graft-versus-host disease after HLA-matched mobilized blood cell transplantation. Blood. 2016; 127: 15028.

20. Floeter AE, McCune JS. Levetiracetam for the prevention of busulfan-induced seizures in pediatric hematopoietic cell transplantation recipients. J Oncol Pharm Pract. 2017; 23: 344-9.

21. Soni S, Skeens M, Termuhlen AM, Bajwa RPS, Gross TG, Pai V. Levetiracetam for busulfan-induced seizure prophylaxis in children undergoing hematopoietic stem cell transplantation. Pediatr Blood Cancer. 2012; 59: 762-4.

22. Akiyama K, Kume T, Fukaya M, Shiki I, Enami T, Tatara R, et al. Comparison of levetiracetam with phenytoin for the prevention of intravenous busulfan-induced seizures in hematopoietic cell transplantation recipients. Cancer Chemother Pharmacol. 2018; 82: 717-21.

23. McCune JS, Wang T, Subait KB, Aljurf M, Beitinjaneh A, Bubalo J, et al. Association of Antiepileptic Medications with Outcomes after Allogeneic Hematopoietic Cell Transplantation with Busulfan/Cyclophosphamide Conditioning. Biol Blood Marrow Transplant. 2019; 25: 1424-31.

24. Palmer J, McCune JS, Perales MA, Marks D, Bubalo J, Mohty $\mathrm{M}$, et al. Personalizing Busulfan-Based Conditioning: Considerations from the American Society for Blood and Marrow Transplantation Practice Guidelines Committee. Biol Blood Marrow Transplant. 2016; 22: 1915-25.

https://doi.org/10.31547/bct-2020-022

Copyright (C)2021 Asia-Pacific Blood and Marrow Transplantation Group (APBMT). This is an open access article distributed under CC BY-NC license (https://creativecommon s.org/licenses/by-nc/4.0/). 